\title{
Electronic excitation spectrum of calcium-doped picene: Electron energy-loss spectroscopy study
}

\author{
Friedrich Roth, ${ }^{1,2}$ Eric Müller, ${ }^{1}$ Bernd Rellinghaus, ${ }^{1}$ Bernd Büchner, ${ }^{1}$ and Martin Knupfer ${ }^{1}$ \\ ${ }^{1}$ IFW Dresden, P.O. Box 270116, D-01171 Dresden, Germany \\ ${ }^{2}$ Center for Free-Electron Laser Science / DESY, Notkestraße 85, D-22607 Hamburg, Germany
}

(Received 7 August 2013; published 6 November 2013)

\begin{abstract}
The electronic excitations of Ca-doped picene films have been investigated using electron energy-loss spectroscopy in transmission. We demonstrate that a composition of $\mathrm{Ca}_{3}$ picene can be achieved by evaporation of $\mathrm{Ca}$ onto pristine picene films under ultrahigh vacuum conditions. The core level excitations indicate a charge transfer of one electron per added $\mathrm{Ca}$ to the picene molecules and a hybridization of $\mathrm{Ca}$ and picene derived electronic states. Upon doping a new spectral feature is observed at about $2 \mathrm{eV}$ in the electronic excitation spectrum. This new feature does not disperse which signals its localized character. The data analysis using a Kramers-Kronig transformation suggests that $\mathrm{Ca}_{3}$ picene has a nonmetallic ground state.
\end{abstract}

DOI: 10.1103/PhysRevB.88.205105

PACS number(s): 79.20.Uv, 78.20.-e, 74.25.Jb

\section{INTRODUCTION}

Molecular materials, in particular $\pi$-conjugated organic compounds, are well-known prototypes of electrical insulators and semiconductors. Their electronic properties are a result of the more delocalized $\pi$ electrons, and their potential application in organic electronic devices has motivated many investigations in the past. ${ }^{1-3}$ One route to change their electronic properties is a reaction with alkali metals, whereby the alkalis intercalate the lattice of the molecular crystal and donate their outer $s$ electron to the molecules. This can lead to novel physical properties, such as metallicity or superconductivity.

The discovery of superconductivity in K-doped picene with a transition temperature up to $18 \mathrm{~K}$ (Ref. 4) might be the starting point for the development of a new class of aromatic hydrocarbon superconductors, comprising molecular crystals doped with alkali or alkaline earth metals. Superconductivity with in part rather high $T_{c}$ 's was also reported in phenanthrene $\left[T_{c}=5 \mathrm{~K}\right.$ (Ref. 5)], coronene $\left[T_{c}=15 \mathrm{~K}\right.$ (Ref. 6)], and 1,2;8,9-dibenzopentacene $\left[T_{c}=33 \mathrm{~K}\right.$ (Ref. 7)] upon $\mathrm{K}$ doping. Especially in the latter case, $T_{c}$ is higher than in any other organic superconductor besides the alkali-metal doped fullerides. ${ }^{8}$ All together all these crystals consist of polycyclic aromatic hydrocarbons, i. e., planar molecules formed by a number of juxtaposed hexagonal benzene rings.

The nature and range of parameters for superconductivity in intercalated hydrocarbons are presently under lively debate. Charge carrier doping is one of the preconditions for the observation of superconductivity. It is, therefore, helpful to investigate the influence of doping on the electronic structure also for other dopands, such as calcium, which can formally contribute two additional electrons to the molecular species. Interestingly, in the case of Ca-intercalated graphite it was found that changing the dopand also affected directly physical properties, such as $T_{c}{ }^{9-11}$

In this contribution we report experimental studies of the influence of $\mathrm{Ca}$ addition on the electronic excitation spectrum of picene using electron energy-loss spectroscopy (EELS). EELS studies of other undoped as well as doped molecular materials in the past have provided useful insight into their electronic properties. ${ }^{12-14}$ We demonstrate that the electronic excitation spectrum significantly changes upon $\mathrm{Ca}$ addition to picene, and we discuss similarities and differences to potassium-intercalated picene.

\section{EXPERIMENT}

Thin films of picene (for a schematic representation of the molecular structure of picene, see inset of Fig. 1) were prepared by thermal evaporation under high vacuum onto single crystalline $\mathrm{KBr}$ substrates kept at room temperature with a deposition rate of $0.2 \mathrm{~nm} / \mathrm{min}$ and an evaporation temperature of about $440 \mathrm{~K}$. The film thickness was about $100 \mathrm{~nm}$. These picene films were floated off in distilled water, mounted onto standard electron microscopy grids, and transferred into the spectrometer. Prior to the EELS measurements the films were characterized in situ using electron diffraction. In Fig. 1 we show the obtained electron diffraction profiles of pristine as well as Ca-doped picene thin films. An analysis of the diffraction peaks of undoped picene leads to the conclusion that our films consist mostly of crystallites with their $a, b$ plane parallel to the film surface. Furthermore, all observed diffraction peaks were consistent with the crystal structure of pristine picene, ${ }^{15}$ similar to our previous experiments on picene. ${ }^{14}$ Upon calcium intercalation the diffraction profile changes in such a way that primarily the intensity of the main diffraction peaks decreases upon calcium addition. Unfortunately, the crystal structure of calcium-doped picene is not known until now. However, from our measurements we can conclude that even after calcium doping, some reflexes at $1.26,1.81$, and $2.5 \AA^{-1}$ can be observed and correspond to diffraction peaks of undoped picene. This leads to the inference that on the one hand the doped films are already crystallized and on the other hand calcium intercalation does not affect the molecule arrangement in the films in a strong manner.

$\mathrm{Ca}$ intercalation was achieved by evaporation of $\mathrm{Ca}$ from a commercial molybdenum crucible (Omicron Nano Technology GmbH, Germany) onto the thin picene films under ultrahigh vacuum conditions (base pressure lower than $10^{-10} \mathrm{mbar}$ ). This has been carried out in several steps until saturation, i. e., until no further stoichiometric change could be observed in the spectra. During Ca evaporation, the films were kept at room temperature. Subsequent annealing of these films 


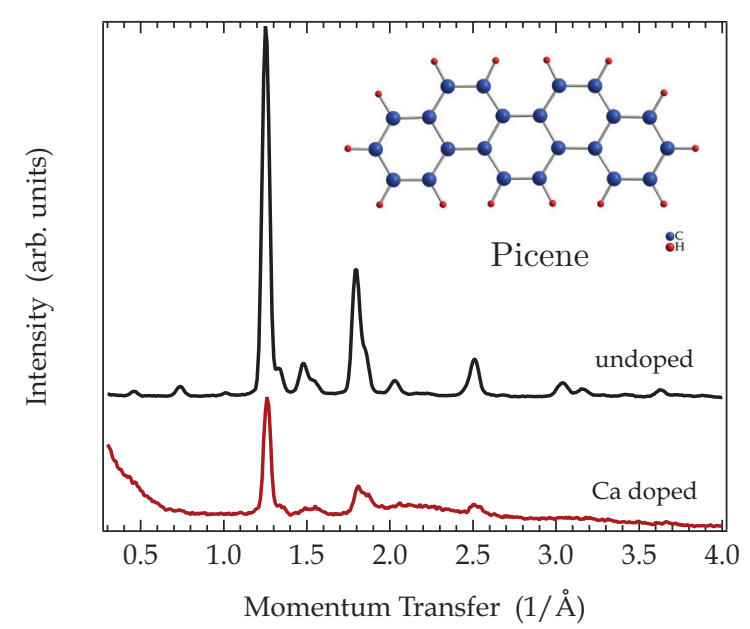

FIG. 1. (Color online) Comparison between the elastic diffraction profiles of pristine as well as Ca-doped picene.

for several hours at $450 \mathrm{~K}$ did not change the stoichiometry or the electronic excitations as obtained in our study.

All electron diffraction studies and loss function measurements were carried out using the $172 \mathrm{keV}$ spectrometer described in detail elsewhere. ${ }^{16}$ The energy and momentum resolution were chosen to be $85 \mathrm{meV}$ and $0.03 \AA^{-1}$, for the loss function measurements and $360 \mathrm{meV}$ and $0.03 \AA^{-1}$ for the core excitations, respectively. We have measured the loss function $\operatorname{Im}[-1 / \epsilon(\mathbf{q}, \omega)]$, which is proportional to the dynamic structure factor $\mathbf{S}(\mathbf{q}, \omega)$, for a momentum transfer $\mathbf{q}$ parallel to the film surface $[\epsilon(\mathbf{q}, \omega)$ is the dielectric function].

\section{RESULTS AND DISCUSSION}

The first direct influence of calcium addition to the electronic structure of the picene samples can be observed in measurements of $\mathrm{C} 1 s$ and $\mathrm{Ca} 2 p$ core level excitations as shown in Fig. 2. Moreover, these data can be used to determine the achieved stoichiometry of the films under investigation by analyzing the relative core level excitation intensities of the two core excitation edges (for details of this procedure, see Refs. 17 and 18). We obtain a composition for our films of about $\mathrm{Ca}_{3}$ picene with an error around $10 \%$. In Fig. 2 we also compare the $\mathrm{C} 1 \mathrm{~s}$ core level spectrum for calcium-doped picene to those of undoped as well as K-doped picene. All three spectra were normalized in the region between 292 and $294 \mathrm{eV}$, i. e., to the $\sigma^{*}$ derived intensity.

Equivalent to other $\pi$ conjugated materials, ${ }^{19-22}$ the $\mathrm{C} 1 s$ edge represents excitations into unoccupied carbon $2 p$ states, and thus allows one to probe the projected unoccupied electronic density of states. In the case of pristine picene the spectrum is dominated by a strong excitation feature at around 284 to $286 \mathrm{eV}$, which can be assigned to transitions into $\pi^{*}$ states representing the unoccupied conduction bands. Upon doping, both with calcium as well as with potassium, a reduction of the spectral weight of this $\mathrm{C} 1 s$ excitation feature can be observed, which signals a successful doping of the films. Remarkably, the decrease in intensity is nearly the same in both cases. Also, the spectral shape of the two curves is very similar, with a small difference at the excitation onset

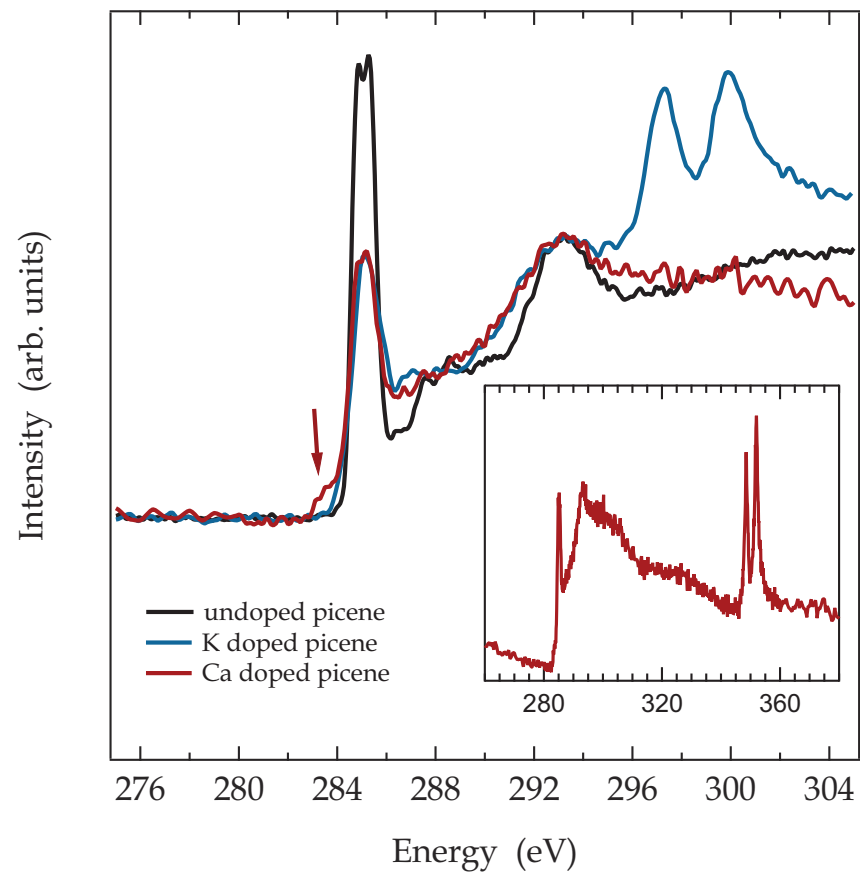

FIG. 2. (Color online) Comparison among the C $1 s$ core level excitations of undoped, potassium as well as calcium-doped picene. The two spectral features at 297 and $300 \mathrm{eV}$ represent excitation from the $\mathrm{K} 2 p$ core levels. The inset shows the data for calciumdoped picene in a wider energy range including the $\mathrm{Ca} 2 p$ core level excitations.

only, where for Ca-doped picene we observe an additional small shoulder (around $283.8 \mathrm{eV}$ ).

The fact that the $\mathrm{C} 1 s$ excitation curves of $\mathrm{K}$ - and Ca-doped picene are so similar clearly indicates that the amount of charge transfer in the two structures is practically the same. Note that the composition of the K-doped picene film is $\mathrm{K}_{3}$ picene, and each potassium provides one electron to the picene molecules. ${ }^{14}$ This, however, seems to be in contradiction to the composition of our Ca-doped picene films of $\mathrm{Ca}_{3}$ picene and the divalent character of $\mathrm{Ca}$. These would suggest a charge transfer of two electrons to picene molecules per $\mathrm{Ca}$, i. e., a doping level that is twice as high as signaled by the $\mathrm{C} 1 s$ core level spectra. On the other hand, it is well known from $\mathrm{Ca}$-doped $\mathrm{C}_{60}$ as well as from $\mathrm{Ca}$-intercalated graphite $\mathrm{CaC}_{6}$, that there is hybridization of $\mathrm{Ca}$ states and those of the counterparts, which leads to the formation of mixed electronic states, and in turn to a reduction of the charge that is transferred to the molecules or graphite planes in comparison to the value of two transferred electrons in a purely ionic picture. ${ }^{23-25}$ This was also observed and calculated for other alkaline-earth-doped compounds, such as Ba-doped $\mathrm{C}_{60}$ or Ba-doped single wall carbon nanotube (SWCNTs). ${ }^{26-29}$ We conclude that this is also the case for $\mathrm{Ca}$-doped picene resulting in an effective charge transfer of only one electron from $\mathrm{Ca}$ to picene in $\mathrm{Ca}_{3}$ picene. The appearance of the small shoulder at the lowest excitation energy in the $\mathrm{C} 1 \mathrm{~s}$ core level excitation spectra (indicated with an arrow in Fig. 2) from these films corroborates this conclusion. Such low energy shoulders have also been observed for $\mathrm{CaC}_{6}$ (Ref. 17) or Ba-doped SWCNTs, ${ }^{29}$ and they represent the 

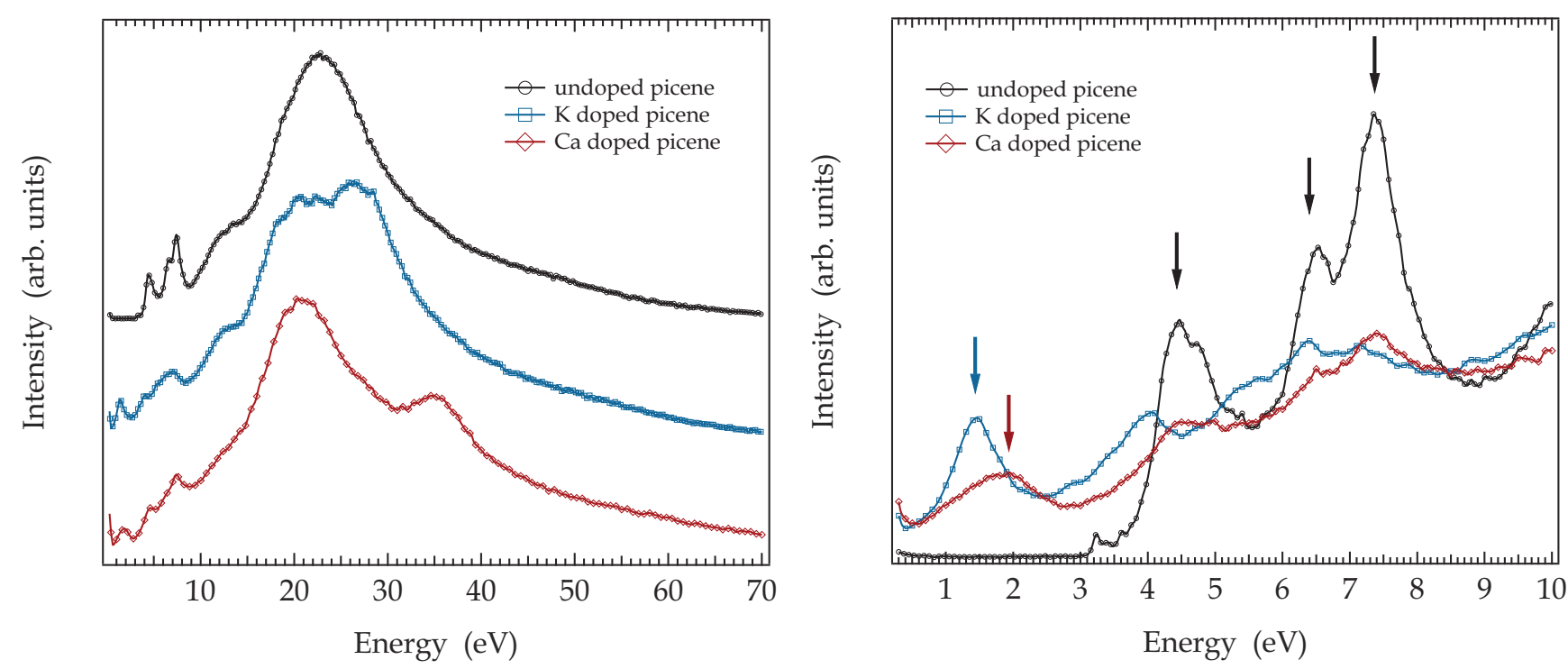

FIG. 3. (Color online) Comparison between the loss functions of pristine picene (black circles) as well as potassium- (blue squares) and calcium-intercalated (red diamonds) picene. All measurements have been done with a momentum transfer of $0.1 \AA^{-1}$.

hybridization induced variations of the unoccupied carbonderived electronic states. ${ }^{23-28}$

Doping of picene also causes major changes in the electronic excitation spectrum as revealed in Fig. 3, where we show a comparison of the measured loss functions of undoped, potassium as well as calcium-doped picene in an energy range between 0 and $70 \mathrm{eV}$ (left panel), and also from 0 to $10 \mathrm{eV}$ (right panel). These data are taken with a small momentum transfer $\mathbf{q}$ of $0.1 \AA^{-1}$, which represents the optical limit. The spectrum of undoped picene is dominated by a broad peak centered at $23 \mathrm{eV}$, which corresponds to the $\pi+\sigma$ plasmon representing a collective oscillation of all valence electrons and additional some low energy features below $10 \mathrm{eV}$. The energy position of the $\pi+\sigma$ plasmon is similar to that observed for other $\pi$ conjugated materials. ${ }^{16,20}$

The situation clearly changes when picene is doped with potassium or calcium. The volume plasmons either shifts to lower energies at around $20 \mathrm{eV}$ (Ca-doped picene) or develops into a double peak feature (K-doped picene). In addition, a new spectral feature at $36 \mathrm{eV}$ for calcium doping can be observed. The peaks that show up upon metal addition can be directly associated to the $\mathrm{K} 3 p$ (around $18 \mathrm{eV}$ ) and $\mathrm{Ca} 3 p$ (at about $36 \mathrm{eV}$ ) core excitation, respectively. This can be seen as an additional evidence of a successful doping of the thin films under investigation.

The changes in the low energy region below $10 \mathrm{eV}$ are more complex. In case of undoped picene (cf. right panel of Fig. 3, black circles) the loss function is characterized by a band gap followed by several maxima (indicated by black arrows), which are due to excitation between the occupied and unoccupied electronic levels. At the excitation onset between 3 and $4 \mathrm{eV}$, small, sharp features arise from singlet excitons in picene. ${ }^{30}$

Doping changes the shape of the spectrum tremendously as can be seen in Fig. 3 (right panel). For potassium doping (blue squares) the former maxima show a downshift, which can be identified as a relaxation of the molecular structure due to the filling of antibonding $\pi^{*}$ levels. In addition, a new feature at about $1.5 \mathrm{eV}$ can be observed (displayed with a blue arrow). This new low energy excitation can be conclusively assigned to the charge carrier plasmon of K-doped picene. ${ }^{14,31}$

For calcium-intercalated picene, we also observe a significant broadening of all spectral features, and again there is a new spectral structure at low energies (centered around $1.9 \mathrm{eV}$ and indicated by a red arrow). Interestingly, a sizable downshift of the excitation maxima as seen in case of $\mathrm{K}$ doping is not observed. We attribute this difference to the hybridization between $\mathrm{Ca}$ and molecular $\pi$ levels (see discussion above), which does not result in a filling of antibonding molecular orbitals alone, but causes further changes of the electronic levels.

The most interesting part of the excitation spectrum as seen in Fig. 3 is the new low energy feature. For K-doped picene $\left(\mathrm{K}_{3}\right.$ picene), this could be assigned to a charge carrier plasmon. ${ }^{14,31}$ This then is in correspondence to the fact that $\mathrm{K}_{3}$ picene should be metallic, since it has been reported to be even a superconductor. For Ca-doped picene information on the ground state is not available yet. We have carried out a Kramers-Kronig analysis (KKA) of the loss function of Ca-doped picene ( $\mathrm{Ca}_{3}$ picene). This allows the determination of the dielectric function of our $\mathrm{Ca}$-doped picene films, and in this way opens further insight into its electronic properties.

In order to be able to carry out this KKA procedure, the measured data have to be corrected for contributions of the elastic line and of multiple scattering effects (for details, see Refs. 16 and 32). The subtraction of the elastic line contributions has been performed using two alternative approaches. We have modeled the measured loss function at low energies either by a simple Drude model or by Lorentz oscillators (see Ref. 32). While the latter would correspond to a nonmetallic electronic ground state, the former represents a metal. Subsequently, we have carried out the KKA, either assuming a metallic ground state or an insulating ground state 


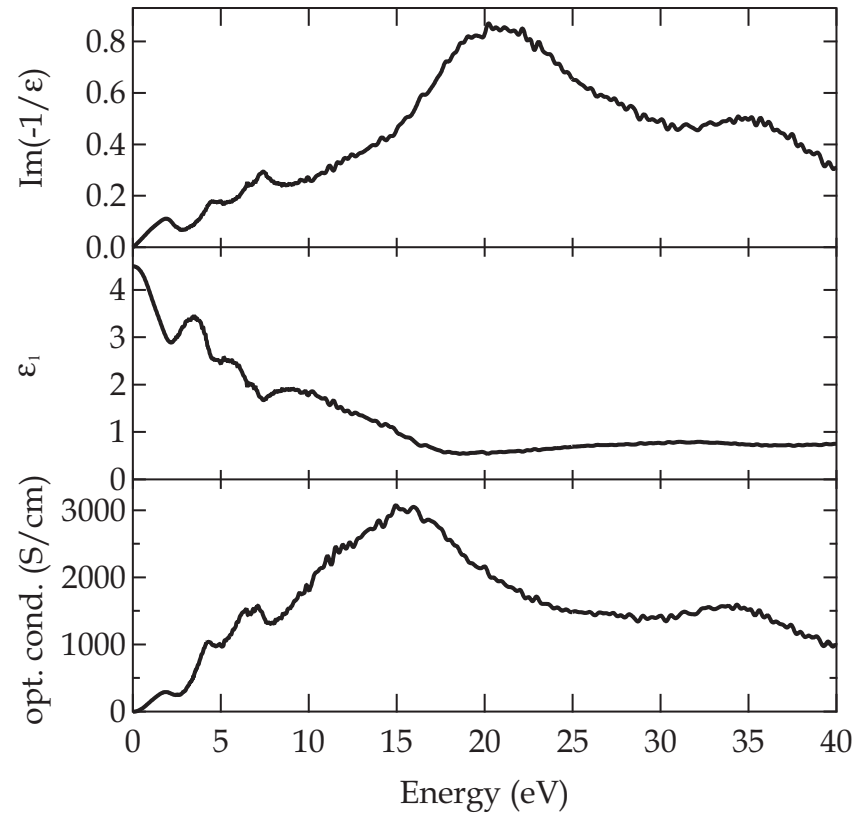

FIG. 4. Loss function $[\operatorname{Im}(-1 / \epsilon)]$, real part $\epsilon_{1}$, and optical conductivity of the dielectric function of Ca-doped picene. The momentum transfer is $q=0.1 \AA^{-1}$. Note that in contrast to Fig. 3, the loss function is corrected for the contribution of the direct beam.

with a finite dielectric constant at zero energy. Finally, we have calculated the optical sum rule for both cases, and compared the resulting values with the number of valence electrons that are expected for $\mathrm{Ca}_{3}$ picene. ${ }^{33}$

The result of our analysis clearly indicates that $\mathrm{Ca}_{3}$ picene cannot be described assuming a metallic ground state. Rather, it is characterized by nonmetallic properties, but with a quite small energy gap, which is suggested by the measured loss function in Fig. 3. In Fig. 4, we summarize the results of the $\mathrm{KKA}$, and we show the loss function (after corrections, see previous paragraph), the resulting real part of the dielectric function $\epsilon_{1}$, and the optical conductivity $\left(\sigma=\omega \times \epsilon_{2}, \epsilon_{2}\right.$ being the imaginary part of the dielectric function). We arrive at a dielectric constant $\epsilon_{1}(0)$ of about 4.5 for our $\mathrm{Ca}_{3}$ picene films, a value that is reasonable for $\pi$ conjugated molecular crystals. Moreover, the optical conductivity as shown in Fig. 4 again indicates that the energy gap of $\mathrm{Ca}_{3}$ picene is rather small, below the resolution that we can achieve in our experiment.

The reasons for the nonmetallic ground state of $\mathrm{Ca}_{3}$ picene can be manifold. We emphasize that the achievement of metallic compounds by intercalation of molecular crystals with alkali metals is an exceptional case. In many cases this has been attributed to the presence of electronic correlation effects in these crystals due to the rather small size of the molecules and the rather weak intermolecular interactions in the condensed phase. A prominent example are the fullerenes, where this has been discussed in detail (e. g., Refs. 20 and 34-37).

Indeed, the importance of electronic correlation effects has also been pointed out for intercalated picene molecular solids in a number of publications. ${ }^{38-42}$ Most importantly, various photoelectron spectroscopy studies revealed that thin films of potassium-intercalated picene show a negligible density of

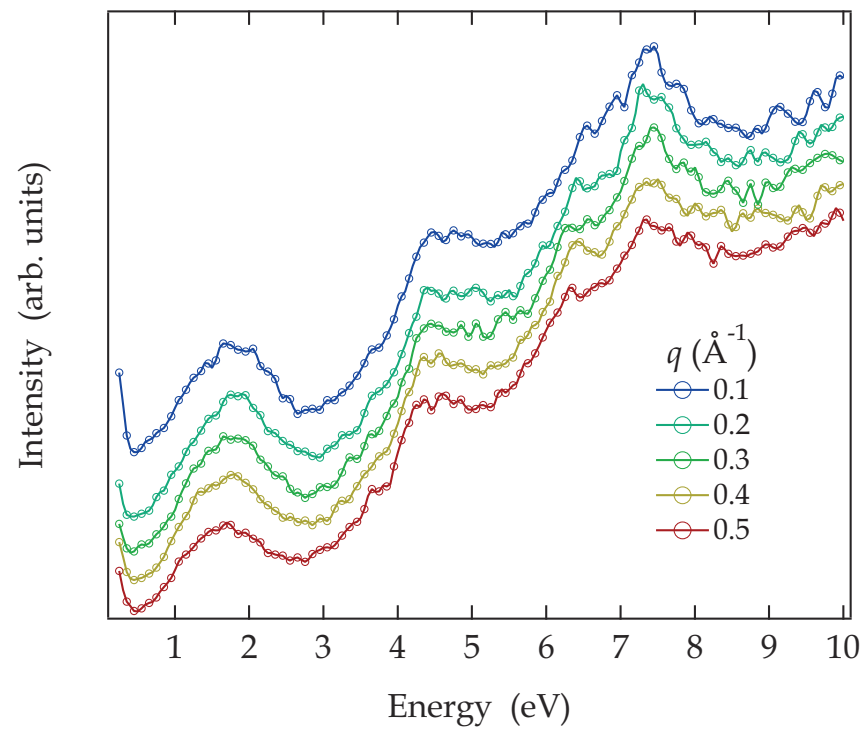

FIG. 5. (Color online) Momentum dependence of calciumintercalated picene in a range between 0.1 and $0.5 \AA^{-1}$. The momentum transfer increases from top to bottom.

states at the Fermi level which has been explained by such correlations effects. ${ }^{40-42}$

It therefore seems to be quite likely that also for $\mathrm{Ca}$ doped picene the electronic correlations are strong enough to localize the charge carriers and to lead to a nonmetallic ground state. Moreover, the fact that there is hybridization between $\mathrm{Ca}$ and picene derived states in $\mathrm{Ca}$-doped picene (see previous discussion) demonstrates that a rigid bandlike behavior upon intercalation and associated charge transfer certainly is not appropriate to describe this compound. Further work is required to fully understand the electronic properties of Ca-doped picene, in particular the determination of the crystal structure of this material is an important ingredient.

Finally, we have also studied the momentum dependence of the lowest electronic excitation of Ca-doped picene within our EELS investigations. In Fig. 5 we show the evolution of the loss function of our $\mathrm{Ca}_{3}$ picene films as a function of momentum transfer up to $0.5 \AA^{-1}$. These data demonstrate that the electronic excitation spectrum of $\mathrm{Ca}_{3}$ picene is rather momentum independent. In other words, the electronic excitations are localized, a situation that is generally quite common in molecular crystals. In particular, also the lowest lying excitation, which has been induced by the $\mathrm{Ca}$ doping process, does not show a dispersion, which is in contrast to the situation in $\mathrm{K}_{3}$ picene, where the doping induced low energy feature showed a negative dispersion, ${ }^{14}$ which could be rationalized in terms of a competition between metallicity and electronic localization on the molecular units. ${ }^{31}$ This underlines the differences in the electronic structure between $\mathrm{Ca}_{3}$ picene and $\mathrm{K}_{3}$ picene as discussed in this paper.

\section{SUMMARY}

To summarize, we have investigated the electronic excitations of Ca-doped picene films using electron energy-loss spectroscopy in transmission. We have shown that a doping 
level of $\mathrm{Ca}_{3}$ picene can be reached by evaporation of $\mathrm{Ca}$ onto the picene films under vacuum conditions. Core level excitation spectra strongly indicate that the charge transfer (doping) to the picene molecules is almost the same as for $\mathrm{K}_{3}$ picene, i.e., each $\mathrm{Ca}$ donates one electron only to the picene molecules. Our data additionally show indications for a hybridization of $\mathrm{Ca}$ and picene derived electronic states. The valence band excitations substantially change as a function of doping. In particular, a new feature appears around $2 \mathrm{eV}$, in the former gap of picene. This doping induced excitation does not show an energy variation upon increasing momentum transfer, demonstrating its localized character. The analysis of the loss function in the valence band region using a Kramers-Kronig analysis indicates that $\mathrm{Ca}_{3}$ picene is characterized by a nonmetallic ground state, which might be caused by a combination of the mentioned hybridization and electronic correlations.

\section{ACKNOWLEDGMENTS}

We thank M. Naumanm, R. Hübel, and S. Leger for technical assistance. This work has been supported by the Deutsche Forschungsgemeinschaft (Grant No. KN393/14).
${ }^{1}$ C. J. Brabec, V. Dyakonov, J. Parisi, and N. S. Sariciftci, Organic Photovoltaics: Concepts and Realization (Springer, Berlin, 2011).

${ }^{2}$ H. Klauk, Organic Electronics: Materials, Manufacturing, and Applications (Wiley-VCH, Weinheim, Germany, 2006).

${ }^{3}$ Z. V. Vardeny, Organic Spintronics (CRC Press, Boca Raton, FL, 2010).

${ }^{4}$ R. Mitsuhashi, Y. Suzuki, Y. Yamanari, H. Mitamura, T. Kambe, N. Ikeda, H. Okamoto, A. Fujiwara, M. Yamaji, N. Kawasaki, Y. Maniwa, and Y. Kubozono, Nature (London) 464, 76 (2010).

${ }^{5}$ X. Wang, R. Liu, Z. Gui, Y. Xie, Y. Yan, J. Ying, X. Luo, and X. Chen, Nat. Commun. 2, 507 (2011).

${ }^{6}$ Y. Kubozono, H. Mitamura, X. Lee, X. He, Y. Yamanari, Y. Takahashi, Y. Suzuki, Y. Kaji, R. Eguchi, K. Akaike, T. Kambe, H. Okamoto, A. Fujiwara, T. Kato, T. Kosugi, and H. Aoki, Phys. Chem. Chem. Phys. 13, 16476 (2011).

${ }^{7}$ M. Xue, T. Cao, D. Wang, Y. Wu, H. Yang, X. Dong, J. He, F. Li, and G. F. Chen, Sci. Rep. 2, 1 (2012).

${ }^{8}$ A. Y. Ganin, Y. Takabayashi, Y. Z. Khimyak, S. Margadonna, A. Tamai, M. J. Rosseinsky, and K. Prassides, Nat. Mater. 7, 367 (2008).

${ }^{9}$ N. Emery, C. Hérold, M. d'Astuto, V. Garcia, C. Bellin, J. F. Marêché, P. Lagrange, and G. Loupias, Phys. Rev. Lett. 95, 087003 (2005)

${ }^{10}$ Y. Koike, S. ichi Tanuma, H. Suematsu, and K. Higuchi, J. Phys. Chem. Solids 41, 1111 (1980).

${ }^{11}$ N. B. Hannay, T. H. Geballe, B. T. Matthias, K. Andres, P. Schmidt, and D. MacNair, Phys. Rev. Lett. 14, 225 (1965).

${ }^{12}$ M. Knupfer, J. Fink, E. Zojer, G. Leising, and D. Fichou, Chem. Phys. Lett. 318, 585 (2000).

${ }^{13}$ F. Roth, R. Schuster, A. König, M. Knupfer, and H. Berger, J. Chem. Phys. 136, 204708 (2012).

${ }^{14}$ F. Roth, B. Mahns, B. Büchner, and M. Knupfer, Phys. Rev. B 83, 144501 (2011).

${ }^{15}$ A. De, R. Ghosh, S. Roychowdhury, and P. Roychowdhury, Acta Crystallogr. C 41, 907 (1985).

${ }^{16}$ J. Fink, Adv. Electr. Electr. Phys. 75, 121 (1989).

${ }^{17}$ F. Roth, A. König, C. Kramberger, T. Pichler, B. Büchner, and M. Knupfer, Europhys. Lett. 102, 17001 (2013).

${ }^{18}$ R. Egerton, Electron Energy-Loss Spectroscopy in the Electron Microscope, 2nd ed. (Plenum Press, New York, 1996).

${ }^{19}$ M. Knupfer, T. Pichler, M. Golden, J. Fink, A. Rinzler, and R. Smalley, Carbon 37, 733 (1999).

${ }^{20}$ M. Knupfer, Surf. Sci. Rep. 42, 1 (2001).
${ }^{21}$ F. Roth, A. König, R. Kraus, M. Grobosch, T. Kroll, and M. Knupfer, Eur. Phys. J. B 74, 339 (2010).

${ }^{22}$ F. Roth, M. Gatti, P. Cudazzo, M. Grobosch, B. Mahns, B. Büchner, A. Rubio, and M. Knupfer, New J. Phys. 12, 103036 (2010).

${ }^{23}$ S. Saito and A. Oshiyama, Solid State Commun. 83, 107 (1992).

${ }^{24}$ Y. Chen, D. M. Poirier, M. B. Jost, C. Gu, T. R. Ohno, J. L. Martins, J. H. Weaver, L. P. F. Chibante, and R. E. Smalley, Phys. Rev. B 46, 7961 (1992).

${ }^{25}$ H. Romberg, M. Roth, and J. Fink, Phys. Rev. B 49, 1427 (1994).

${ }^{26}$ S. Saito and A. Oshiyama, Phys. Rev. Lett. 71, 121 (1993).

${ }^{27}$ S. C. Erwin and M. R. Pederson, Phys. Rev. B 47, 14657 (1993).

${ }^{28}$ M. Knupfer, F. Stepniak, and J. H. Weaver, Phys. Rev. B 49, 7620 (1994).

${ }^{29}$ X. Liu, T. Pichler, M. Knupfer, and J. Fink, Phys. Rev. B 70, 245435 (2004).

${ }^{30}$ F. Roth, B. Mahns, B. Büchner, and M. Knupfer, Phys. Rev. B 83, 165436 (2011)

${ }^{31}$ P. Cudazzo, M. Gatti, F. Roth, B. Mahns, M. Knupfer, and A. Rubio, Phys. Rev. B 84, 155118 (2011).

${ }^{32}$ R. Schuster, R. Kraus, M. Knupfer, H. Berger, and B. Büchner, Phys. Rev. B 79, 045134 (2009).

${ }^{33}$ As the crystal structure for $\mathrm{Ca}_{3}$ picene is not known, we have used the unit cell volume for undoped picene. This will cause some uncertainties within our analysis, but it does not affect the conclusions drawn on the nonmetallic ground state of $\mathrm{Ca}_{3}$ picene.

${ }^{34}$ O. Gunnarson, Alkali Doped Fullerides (World Scientific, Singapore, 2004).

${ }^{35}$ R. W. Lof, M. A. van Veenendaal, B. Koopmans, H. T. Jonkman, and G. A. Sawatzky, Phys. Rev. Lett. 68, 3924 (1992).

${ }^{36}$ P. J. Benning, F. Stepniak, and J. H. Weaver, Phys. Rev. B 48, 9086 (1993).

${ }^{37}$ M. Knupfer, D. M. Poirier, and J. H. Weaver, Phys. Rev. B 49, 8464 (1994).

${ }^{38}$ G. Giovannetti and M. Capone, Phys. Rev. B 83, 134508 (2011).

${ }^{39}$ M. Kim, B. I. Min, G. Lee, H. J. Kwon, Y. M. Rhee, and J. H. Shim, Phys. Rev. B 83, 214510 (2011).

${ }^{40}$ B. Mahns, F. Roth, and M. Knupfer, J. Chem. Phys. 136, 134503 (2012).

${ }^{41}$ M. Caputo, G. Di Santo, P. Parisse, L. Petaccia, L. Floreano, A. Verdini, M. Panighel, C. Struzzi, B. Taleatu, C. Lal, and A. Goldoni, J. Phys. Chem. C 116, 19902 (2012).

${ }^{42}$ A. Ruff, M. Sing, R. Claessen, H. Lee, M. Tomić, H. O. Jeschke, and R. Valentí, Phys. Rev. Lett. 110, 216403 (2013). 